\title{
Subjective Health and Happiness in the United States: Gender Differences in the Effects of Socioeconomic Status Indicators
}

\author{
Najmeh Maharlouei ${ }^{1,3}$, Sharon Cobb ${ }^{2}$, Mohsen Bazargan ${ }^{3,4}$, Shervin Assari ${ }^{3 *}$ \\ 'Health Policy Research Center, Institute of Health, Shiraz University of Medical Sciences, Shiraz, Iran \\ ${ }^{2}$ School of Nursing, Charles R Drew University of Medicine and Science, Los Angeles, CA \\ ${ }^{3}$ Department of Family Medicine, Charles R Drew University of Medicine and Science, Los Angeles, CA \\ ${ }^{4}$ Department of Family Medicine, UCLA, Los Angeles, CA
}

Article Info

\section{Article Notes}

Received: March 26, 2020

Accepted: May 14, 2020

\section{*Correspondence:}

Dr. Shervin Assari, MD MPH, Department of Family Medicine, Charles R Drew University of Medicine and Science, Los Angeles, California, USA; Email: shervinassari@cdrewu.edu, assari@umich.edu.

(c) 2020 Assari S. This article is distributed under the terms of the Creative Commons Attribution 4.0 International License.

\section{Keywords}

Socioeconomic position

Socioeconomic status

Education

Self-rated health

Happiness

\section{Abstract}

Background: Education, employment, and marital status are among the main socioeconomic status (SES) indicators that are associated with subjective health and happiness. The effects of these SES indicators may, however, be different for various demographic groups.

Aims: To understand if SES indicators differently impact men and women, we tested gender differences in the effects of education, employment, and marital status on the subjective health and happiness of American adults.

Methods: This cross-sectional study used data of the General Social Survey (GSS), a series of nationally representative surveys between 1972 and 2018 in the US. Our analytical sample included 65,814 adults. The main independent variables were education attainment, marital status, and employment. Outcomes were self-rated health (SRH) and happiness measured using single items. Age and year of the study were covariates. Gender was the moderator.

Results: Overall, high education, being employed, and being married were associated with better SRH and happiness. We, however, found significant interactions between gender and educational attainment, marital status, and employment on the outcomes, which suggested that the effect of high education and marital status were stronger for women. In comparison, the effect of employment was stronger for men. Some inconsistencies in the results were observed for SRH compared to happiness.

Conclusions: In the United States, while education, employment, and marital status are critical social determinants of subjective health and happiness, these effects vary between women and men. Men's outcomes seem to be more strongly shaped by employment, while women's outcomes are more strongly shaped by education and marital status.

\section{Background}

The topic of well-being, that includes subjective health and happiness, has attracted the attention of governments, sociologists, psychologists, and economists ${ }^{1,2}$. There is ample evidence showing a robust relationship between well-being and subjective health ${ }^{1,2}$. At the national level, gross domestic product (GDP) is a robust measure of economic wellbeing. However, it is not ideal because it does not provide a tool to compare other aspects of well-being of demographic sub-groups within a single country. In addition, this measure does not reflect inequalities in subjective health and happiness due to differences in socioeconomic resources such as education, employment, and marital status. As a result, it is essential to consider subjective components of well-being ${ }^{2}$. Hence, selfreported happiness and health, are now being recognized as two 
critical components of well-being that can inform public policies to promote equality and reduce disparities in wellbeing across sub-populations ${ }^{1,3,4}$.

High levels of subjective health and happiness reflect high levels of productivity at all levels, from individuals to the society $3,5,6$. Healthy and happy individuals are more productive and better contribute to the economic progress and prosperity of the country. Healthy and happy individuals also report better inter-personal relationships, which is closely linked to positive physical and mental health ${ }^{3,5}$

Happiness is associated with optimism and enjoyment, and is a potent propelling force providing energy and enthusiasm for individuals and communities ${ }^{7}$. Happy people are better able to combat disease and hence tend to live longer ${ }^{7}$. Happiness boosts the immune system ${ }^{8}$ and increases resilience in the face of adverse economic and social conditions in daily life $\mathrm{e}^{3,6}$. Happier societies also have a lower rate of unnecessary visits to the doctors and hospitals, thereby reducing healthcare burden and costs 9 . They also have less crime and social disorder ${ }^{10}$.

To maximize the average/overall level of the wellbeing of the citizens of each country and to minimize the inequalities and gaps in well-being of sections of their societies, policymakers require local knowledge on the distribution of subjective health and happiness in the subsections of their citizens. This knowledge can determine the special needs of each subsection of the society and can help policymakers with the development of social and health programs and interventions to effectively enhance the well-being of their citizens. Another benefit is determining the distribution of well-being across groups with various degrees of access to social determinants of health. In order to equalize population wellbeing, it is essential to know how each subsection of society is responding to each of the social determinants of health ${ }^{11}$.

Self-Rated Health (SRH) is a reliable and feasible screening tool for detecting people who are going to use public health services ${ }^{4}$. It is a simple measure, and one that is widely used for determining people's health status and for predicting mortality ${ }^{12-14}$. Belichick et al. showed that SRH has a robust consistency across different regions of the US in ascertaining health status and five-year mortality. They also showed that regional variation of reported $\mathrm{SRH}$ is due to real differences in the health status of the inhabitants of the various regions ${ }^{15}$.

Single item questions, including SRH and happiness (the key elements of well-being), provide an excellent, affordable, and quick opportunity to evaluate variation of well-being across populations. These single-item measures are inexpensive, highly reliable, and valid. These tools have provided researchers and policymakers with reliable methods for estimating population well-being ${ }^{2}$. As a result, SRH and happiness items are being included in the national surveys of many countries so that public, social, and health policymakers can use the data for improving citizens' well-being.

Social determinants of health are among the root and fundamental causes of subjective health and happiness of populations. Research has shown that education, income, employment, and marital status influence both SRH and happiness. However, these effects are shown to differ in sub-populations ${ }^{16}$. For example, groups based on race $^{17}$, ethnicity ${ }^{18,19}$, and sexual orientation ${ }^{20}$ may be differently influenced by each of these resources.

A major factor that alters the effects of social determinants on subjective health and happiness is gender ${ }^{21-23}$. Health and happiness may have different effects on men and women, depending on education, employment, and marital status ${ }^{21-29}$. For example, recent research on "the sponge hypothesis" suggests that subjective health measures may function as a sponge ${ }^{30}$, and as a result may reflect factors other than health for women. As a result, the clinical utility and meaning of subjective health may differ for men and women ${ }^{31}$.

\section{Aims}

To better understand the role of gender (as a social rather than as a biological factor) in alerting the relevance of three SES indicators to subjective health and happiness of American adults, we compared American men and women for the effects of educational attainment, employment, and marital status on subjective health and happiness. To produce generalizable results, we used a nationally representative sample.

\section{Methods}

\section{Design and Setting}

The General Social Survey (GSS; 1972-2018) is a stateof-the-art social survey of American adults. The GSS is conducted annually from 1972 to 2018 by the University of Chicago to monitor societal change and social trends of American society over time. The GSS is mainly funded by the National Science Foundation (NSF).

\section{Ethics}

The GSS study protocol is approved by the University of Chicago Institutional Review Board (IRB). All GSS participants have provided informed consent. However, the current study was not deemed to be human subjects research, since all the data were public and fully anonymous.

\section{General Social Survey (GSS)}

The GSS gathers extensive data on the social aspects of contemporary American society. This study has continued 
to monitor the trends of attitudes, behaviors, and beliefs of American adults for over four decades since 1972. The GSS helps us understand how US society and its demographic subgroups have changed over that time. This study provides a unique opportunity to study population variation based on gender, race/ethnicity, and class. Some of the variables cover various aspects of sociology, economics, policymakers, and demography, among others.

\section{Analytical Sample}

The current study included all adults who had participated in the GSS from 1974 to 2018, which included 64,814 individuals.

\section{Study Measures}

Study variables included educational attainment, employment status, marital status, year of survey, gender, race/ethnicity, age, subjective health, and happiness.

\section{Independent variable}

Educational Attainment: Educational attainment was measured as years of schooling, varying from 0 to 20 . It was treated as an interval measure (a higher score reflecting higher education attainment in terms of years of schooling).

Employment: Employment was measured as an ordinal variable, with categories: 1) Working Full-time, 2) Working Part-time, 3) Temporarily Not Working, 4) Unemployed, Laid Off, 5) Retired, 6) School, 7) Keeping House, and 8) Other. We recoded this variable as a binary variable with working full-time as one and any other status as the reference group.

Marital Status: Marital status was assessed as a binary variable: 1) Married versus others including widowed, divorced, separated, and never married. Married was coded as one, and others were the reference category (coded as 0 ).

\section{Outcomes}

Subjective Health: In this study subjective health was measured using a single item Self-Rated Health (SRH) measure. The GSS SRH was a 4-level categorical variable. The exact item was, "Would you say your health, in general, is excellent, good, fair, or poor?" Items were excellent, good, fair, or poor. We merged excellent and good as healthy, and fair or poor as unhealthy. This variable was operationalized as a categorical (binary) variable with SRH ( 1 good, 0 poor/ fair health). A shown by Idler and many others, SRH is a strong predictor of mortality ${ }^{14}$.

Happiness: Happiness was measured using a single item. The item measures general happiness and reads as: "Taken all together, how would you say things are these days - would you say that you are very happy, pretty happy, or not too happy?" Responses were 1) very happy, 2) pretty happy, and 3) not too happy. The item was asked from 1972 to 2010. This variable was operationalized as a categorical (binary) variable (1 being very happy / pretty happy, 0 not too happy).

\section{Covariates}

Age (years), race/ethnicity, and year of the survey were the study covariates. Age was an interval variable, measured in years. Self-identified race and ethnicity were measured as a three-level categorical variable: Whites 0 [the reference group], Blacks 1, and other race/ethnic groups 2 . The Year of the study was operationalized as an interval variable ranging from 1972 to 2018 (Table 1).

\section{Moderator}

Gender: Gender (men 1, women 0) was the moderating factor (effect modifier).

Appendix 1. Frequency of participants based on year in the General Social Survey (GSS) overall and by gender.

\begin{tabular}{|c|c|c|c|c|c|c|}
\hline \multirow[t]{2}{*}{ Year } & \multicolumn{2}{|c|}{ All } & \multicolumn{2}{|c|}{ Men } & \multicolumn{2}{|c|}{ Women } \\
\hline & $\mathrm{n}$ & $\%$ & $n$ & $\%$ & $n$ & $\%$ \\
\hline 1972 & 1613 & 2.5 & 807 & 2.8 & 806 & 2.2 \\
\hline 1973 & 1504 & 2.3 & 701 & 2.4 & 803 & 2.2 \\
\hline 1974 & 1484 & 2.3 & 691 & 2.4 & 793 & 2.2 \\
\hline 1975 & 1490 & 2.3 & 670 & 2.3 & 820 & 2.3 \\
\hline 1976 & 1499 & 2.3 & 669 & 2.3 & 830 & 2.3 \\
\hline 1977 & 1530 & 2.4 & 693 & 2.4 & 837 & 2.3 \\
\hline 1978 & 1532 & 2.4 & 643 & 2.2 & 889 & 2.5 \\
\hline 1980 & 1468 & 2.3 & 641 & 2.2 & 827 & 2.3 \\
\hline 1982 & 1860 & 2.9 & 779 & 2.7 & 1081 & 3.0 \\
\hline 1983 & 1599 & 2.5 & 690 & 2.4 & 909 & 2.5 \\
\hline 1984 & 1473 & 2.3 & 598 & 2.1 & 875 & 2.4 \\
\hline 1985 & 1534 & 2.4 & 688 & 2.4 & 846 & 2.3 \\
\hline 1986 & 1470 & 2.3 & 621 & 2.2 & 849 & 2.3 \\
\hline 1987 & 1819 & 2.8 & 778 & 2.7 & 1041 & 2.9 \\
\hline 1988 & 1481 & 2.3 & 638 & 2.2 & 843 & 2.3 \\
\hline 1989 & 1537 & 2.4 & 660 & 2.3 & 877 & 2.4 \\
\hline 1990 & 1372 & 2.1 & 604 & 2.1 & 768 & 2.1 \\
\hline 1991 & 1517 & 2.3 & 636 & 2.2 & 881 & 2.4 \\
\hline 1993 & 1606 & 2.5 & 685 & 2.4 & 921 & 2.5 \\
\hline 1994 & 2992 & 4.6 & 1290 & 4.5 & 1702 & 4.7 \\
\hline 1996 & 2904 & 4.5 & 1285 & 4.5 & 1619 & 4.5 \\
\hline 1998 & 2832 & 4.4 & 1232 & 4.3 & 1600 & 4.4 \\
\hline 2000 & 2817 & 4.3 & 1229 & 4.3 & 1588 & 4.4 \\
\hline 2002 & 2765 & 4.3 & 1228 & 4.3 & 1537 & 4.2 \\
\hline 2004 & 2812 & 4.3 & 1280 & 4.5 & 1532 & 4.2 \\
\hline 2006 & 4510 & 7.0 & 2003 & 7.0 & 2507 & 6.9 \\
\hline 2008 & 2023 & 3.1 & 930 & 3.3 & 1093 & 3.0 \\
\hline 2010 & 2044 & 3.2 & 891 & 3.1 & 1153 & 3.2 \\
\hline 2012 & 1974 & 3.0 & 885 & 3.1 & 1089 & 3.0 \\
\hline 2014 & 2538 & 3.9 & 1141 & 4.0 & 1397 & 3.9 \\
\hline 2016 & 2867 & 4.4 & 1276 & 4.5 & 1591 & 4.4 \\
\hline 2018 & 2348 & 3.6 & 1052 & 3.7 & 1296 & 3.6 \\
\hline
\end{tabular}




\section{Statistical Analysis}

Data were analyzed using SPSS 22.0 We reported frequency (\%) and mean (standard error; SE) to describe our participants overall as well as by gender. We used Chisquare or independent t-tests to compare men and women for our study variables. Overall, we ran four models for each outcome. For consistency and ease of interpretation, the positive meaning was the outcome. That is happiness ( 1 being very happy / pretty happy, 0 not too happy), and subjective health ( 1 good, 0 poor/fair health) were the primary outcomes (dependent variable). Models 1 and 2 mentioned in tables 2 and 3 were performed in the pooled sample. Model 1 only had the main effects. Model 2, however, also included the gender by educational attainment, marital status, and employment, interaction term. Logistic regression was applied to define the determinant factors of subjective health and happiness in men and women. Educational attainment (years of education), employment status, and marital status were the primary predictors (independent variables), age, and year of the survey were the covariates. Gender was the moderator. Odds Ratio (OR), $\mathrm{SE}, 95 \% \mathrm{CI}$, and $\mathrm{p}$ values were reported.

\section{Results}

\section{Descriptive Statistics}

Sixty-five thousand eight hundred fourteen adults were sampled between 1972 and 2018 (Appendix 1). Loss to follow up for each year was about $2.5 \%$. More than half of the participants were women $(n=36,200 ; 55.9 \%)$. Most participants were White (80.3\%). Men and women differed in employment, marital status, subjective health, and mean age and education, but not happiness (Table 1).

\section{Multivariable models (Outcome: Subjective Health)}

Table 2 presents the summary of the results of our two logistic regression models with educational attainment, employment, and marital status as the independent variables, and subjective health as the dependent variable. Both models were estimated in the overall sample. Model 1 only entered the main effects of SES indicators (educational attainment, employment, and marital status) while controlling for gender and covariates. Model 2 added three interaction terms between gender and our SES indicators.

In Model 1, female gender, being married, white race, younger age, higher educational attainment, and having a fulltime job were associated with better subjective health. Model 2 showed two statistically significant interactions between gender and educational attainment and marital status on subjective health, suggesting that high educational attainment and marital status both have more substantial effects on the subjective health of women than men (Table 2).

Table 1. Descriptive statistics in the overall sample.

\begin{tabular}{|c|c|c|c|c|c|c|}
\hline & \multicolumn{2}{|c|}{ All } & \multicolumn{2}{|c|}{ Men } & \multicolumn{2}{|c|}{ Women } \\
\hline & $\mathbf{n}$ & $\%$ & $\mathbf{n}$ & $\%$ & $\mathbf{n}$ & $\%$ \\
\hline \multicolumn{7}{|l|}{ Gender } \\
\hline Men & 28614 & 44.1 & 28614 & 100.00 & - & - \\
\hline Women & 36200 & 55.9 & - & - & 36200 & 100.00 \\
\hline \multicolumn{7}{|l|}{ Race } \\
\hline White & 52033 & 80.3 & 23408 & 81.8 & 28625 & 79.1 \\
\hline Black & 9187 & 14.2 & 3528 & 12.3 & 5659 & 15.6 \\
\hline Other Race/Ethnic Groups & 3594 & 5.5 & 1678 & 5.9 & 1916 & 5.3 \\
\hline \multicolumn{7}{|l|}{ Employed* } \\
\hline No & 32922 & 50.8 & 10869 & 38.0 & 22053 & 60.9 \\
\hline Yes & 31892 & 49.2 & 17745 & 62.0 & 14147 & 39.1 \\
\hline \multicolumn{7}{|l|}{ Marital Status* } \\
\hline No & 30685 & 47.3 & 12378 & 43.3 & 18307 & 50.6 \\
\hline Yes & 34129 & 52.7 & 16236 & 56.7 & 17893 & 49.4 \\
\hline \multicolumn{7}{|l|}{ Subjective Health $* \mathrm{~m}$} \\
\hline Not Healthy & 11845 & 24.9 & 4913 & 23.3 & 6932 & 26.2 \\
\hline Healthy & 35745 & 75.1 & 16185 & 76.7 & 19560 & 73.8 \\
\hline \multicolumn{7}{|l|}{ Happiness } \\
\hline Not Happy & 7668 & 12.8 & 3356 & 12.7 & 4312 & 12.9 \\
\hline \multirow[t]{2}{*}{ Happy } & 52386 & 87.2 & 23159 & 87.3 & 29227 & 87.1 \\
\hline & Mean & SD & Mean & SD & Mean & SD \\
\hline Age (Years)* & 45.86 & 17.28 & 45.23 & 16.88 & 46.35 & 17.58 \\
\hline Educational Attainment $(0-20)^{*}$ & 7.574 & 6.09 & 7.87 & 6.09 & 7.34 & 6.08 \\
\hline
\end{tabular}

m: 17224 missing cases

${ }^{*} p<0.05$ for comparison of men and women

SD: Standard Deviation 
Table 2. Summary of logistic regressions on subjective health in the pooled sample.

\begin{tabular}{|c|c|c|c|c|c|c|c|c|c|c|c|c|}
\hline \multirow[b]{3}{*}{ Gender (Women) } & \multicolumn{6}{|c|}{ Model 1} & \multicolumn{6}{|c|}{ Model 2} \\
\hline & \multirow{2}{*}{$\begin{array}{c}b \\
0.09\end{array}$} & \multirow{2}{*}{$\begin{array}{c}\text { SE } \\
0.02\end{array}$} & \multirow{2}{*}{$\frac{\mathrm{OR}}{1.10}$} & \multicolumn{2}{|c|}{$95 \% \mathrm{Cl}$} & \multirow{2}{*}{$\begin{array}{c}\mathrm{p} \\
.000\end{array}$} & \multirow{2}{*}{$\begin{array}{c}b \\
-0.01\end{array}$} & \multirow{2}{*}{$\begin{array}{c}\text { SE } \\
0.04\end{array}$} & \multirow{2}{*}{$\frac{\text { OR }}{0.99}$} & \multicolumn{2}{|c|}{$95 \% \mathrm{Cl}$} & \multirow{2}{*}{$\begin{array}{c}\mathrm{P} \\
.767\end{array}$} \\
\hline & & & & 1.05 & 1.15 & & & & & 0.91 & 1.08 & \\
\hline Race/ethnicity (Black) & -0.36 & 0.03 & 0.70 & 0.66 & 0.74 & .000 & -0.35 & 0.03 & 0.70 & 0.66 & 0.75 & .000 \\
\hline Race/ethnicity (Other) & -0.39 & 0.05 & 0.68 & 0.62 & 0.75 & .000 & -0.39 & 0.05 & 0.68 & 0.62 & 0.75 & .000 \\
\hline Age (Years) & -0.02 & 0.00 & 0.98 & 0.98 & 0.98 & .000 & -0.02 & 0.00 & 0.98 & 0.98 & 0.98 & .000 \\
\hline Time (Year) & 0.00 & 0.00 & 1.00 & 1.00 & 1.00 & .007 & 0.00 & 0.00 & 1.00 & 1.00 & 1.00 & .009 \\
\hline Educational Attainment (0-20) & 0.05 & 0.00 & 1.05 & 1.04 & 1.05 & .000 & 0.04 & 0.00 & 1.04 & 1.03 & 1.05 & .000 \\
\hline Fulltime Employment & 0.75 & 0.02 & 2.11 & 2.01 & 2.22 & .000 & 0.80 & 0.04 & 2.22 & 2.07 & 2.38 & .000 \\
\hline Marital Status (Married) & 0.30 & 0.02 & 1.35 & 1.29 & 1.41 & .000 & 0.21 & 0.04 & 1.23 & 1.15 & 1.32 & .000 \\
\hline Educational Attainment (0-20) x Gender (Women) & & & & & & & 0.01 & 0.00 & 1.01 & 1.00 & 1.02 & .013 \\
\hline Fulltime Employment x Gender (Women) & & & & & & & -0.07 & 0.05 & 0.93 & 0.85 & 1.03 & .152 \\
\hline Marital Status (Married) x Gender (Women) & & & & & & & 0.15 & 0.05 & 1.16 & 1.05 & 1.27 & .002 \\
\hline Constant & 1.32 & 0.05 & 3.74 & & & .000 & 1.36 & 0.05 & 3.90 & & & .000 \\
\hline
\end{tabular}

$\mathrm{Cl}$ : Confidence Interval; SE: Standard

Error; OR: Odds Ratio

Table 3. Summary of logistic regressions on happiness in the pooled sample.

\begin{tabular}{|c|c|c|c|c|c|c|c|c|c|c|c|c|}
\hline \multirow[b]{3}{*}{ Gender (Women) } & \multicolumn{6}{|c|}{ Model 1} & \multicolumn{6}{|c|}{ Model 2} \\
\hline & \multirow{2}{*}{$\begin{array}{c}\mathrm{b} \\
0.18\end{array}$} & \multirow{2}{*}{$\begin{array}{c}\text { SE } \\
0.03\end{array}$} & \multirow{2}{*}{$\begin{array}{c}\text { OR } \\
1.20\end{array}$} & \multicolumn{2}{|c|}{$95 \% \mathrm{Cl}$} & \multirow{2}{*}{$\begin{array}{c}\mathrm{p} \\
.000\end{array}$} & \multirow{2}{*}{$\begin{array}{c}b \\
0.22\end{array}$} & \multirow{2}{*}{$\begin{array}{c}\text { SE } \\
0.05\end{array}$} & \multirow{2}{*}{$\begin{array}{c}\text { OR } \\
1.25\end{array}$} & \multicolumn{2}{|c|}{$95 \% \mathrm{Cl}$} & \multirow{2}{*}{$\begin{array}{c}\mathrm{P} \\
.000\end{array}$} \\
\hline & & & & 1.14 & 1.26 & & & & & 1.14 & 1.37 & \\
\hline Race/ethnicity (Black) & -0.47 & 0.03 & 0.63 & 0.59 & 0.67 & .000 & -0.46 & 0.03 & 0.63 & 0.59 & 0.67 & .000 \\
\hline Race/ethnicity (Other) & -0.27 & 0.05 & 0.76 & 0.69 & 0.85 & .000 & -0.27 & 0.05 & 0.76 & 0.69 & 0.85 & .000 \\
\hline Age (Years) & 0.00 & 0.00 & 1.00 & 1.00 & 1.00 & .081 & 0.00 & 0.00 & 1.00 & 1.00 & 1.00 & .031 \\
\hline Time (Year) & 0.00 & 0.00 & 1.00 & 1.00 & 1.00 & .763 & 0.00 & 0.00 & 1.00 & 1.00 & 1.00 & .707 \\
\hline Educational Attainment (0-20) & 0.03 & 0.00 & 1.03 & 1.03 & 1.04 & .000 & 0.03 & 0.00 & 1.03 & 1.03 & 1.04 & .000 \\
\hline Fulltime Employment & 0.46 & 0.03 & 1.58 & 1.50 & 1.67 & .000 & 0.58 & 0.04 & 1.78 & 1.65 & 1.93 & .000 \\
\hline Marital Status (Married) & 0.93 & 0.03 & 2.54 & 2.40 & 2.67 & .000 & 0.85 & 0.04 & 2.34 & 2.16 & 2.53 & .000 \\
\hline Educational Attainment (0-20) x Gender (Women) & & & & & & & 0.00 & 0.00 & 1.00 & 0.99 & 1.01 & .904 \\
\hline Fulltime Employment x Gender (Women) & & & & & & & -0.21 & 0.05 & 0.81 & 0.73 & 0.90 & .000 \\
\hline Marital Status (Married) x Gender (Women) & & & & & & & 0.13 & 0.05 & 1.14 & 1.03 & 1.27 & .014 \\
\hline Constant & 1.00 & 0.05 & 2.71 & & & .000 & 0.95 & 0.06 & 2.59 & & & .000 \\
\hline
\end{tabular}

$\mathrm{Cl}$ : Confidence Interval; SE: Standard Error;

OR: Odds Ratio

\section{Multivariable models (Outcome: happiness)}

Table 3 presents the summary of the results of our two logistic regression models with educational attainment, employment and marital status as the independent variables, and happiness as the dependent variable. Both models were estimated in the overall sample. Model 1 only entered the main effects of educational attainment, employment, and marital status while controlling for gender and covariates. Model 2 added three interaction terms between gender and 1) educational attainment, 2) employment, and 3) marital status.

Based on Model 1, the female gender, being married, white race, higher educational attainment, high educational attainment, and having a fulltime job were associated with higher levels of happiness. Model 2 showed statistically significant interactions between gender and employment and marital status on happiness, suggesting that the effect of employment is larger for men while the effect of marital status is larger for women (Table 3 ).

\section{Multivariable models (gender-specific results)}

Table 4 and Table 5 present the summary of the results of gender-stratified logistic regression models that test the combined effects of educational attainment, employment, and marital status on subjective health and happiness. Table 4 presents the results for subjective health, and Table 5 presents the results for happiness. The model on the left was performed on men, and the model on the right was performed on women. While in both genders, race, age, educational attainment, employment, and marital status had significant effects on subjective health, the magnitude of the effect of race, educational attainment and marital status was larger for women than for men. By contrast, the magnitude of the effect of employment was larger for men than for women. Race, age, educational attainment, employment, and marital status had significant effects on happiness for both men and women. However, the magnitude of the effect of race, and marital status was larger for women than for men, while the magnitude of the effect of educational attainment and employment was larger for men than for women. 
Table 4. Summary of logistic regressions on subjective health in men and women.

\begin{tabular}{|c|c|c|c|c|c|c|c|c|c|c|c|c|}
\hline \multirow[b]{3}{*}{ Race/ethnicity (Black) } & \multicolumn{6}{|c|}{ Men } & \multicolumn{6}{|c|}{ Women } \\
\hline & \multirow{2}{*}{$\begin{array}{c}b \\
-0.30\end{array}$} & \multirow{2}{*}{$\begin{array}{c}\text { SE } \\
0.05\end{array}$} & \multirow{2}{*}{$\begin{array}{c}\text { OR } \\
0.74\end{array}$} & \multicolumn{2}{|c|}{$95 \% \mathrm{Cl}$} & \multirow{2}{*}{$\begin{array}{c}\mathrm{p} \\
.000\end{array}$} & \multirow{2}{*}{$\begin{array}{c}b \\
-0.38\end{array}$} & \multirow{2}{*}{$\begin{array}{c}\text { SE } \\
0.04\end{array}$} & \multirow{2}{*}{$\begin{array}{c}\text { OR } \\
0.68\end{array}$} & \multicolumn{2}{|c|}{$95 \% \mathrm{Cl}$} & \multirow{2}{*}{$\begin{array}{c}P \\
.000 \\
\end{array}$} \\
\hline & & & & 0.67 & 0.82 & & & & & 0.63 & 0.74 & \\
\hline Race/ethnicity (Other) & -0.36 & 0.07 & 0.70 & 0.61 & 0.81 & .000 & -0.41 & 0.07 & 0.66 & 0.58 & 0.76 & .000 \\
\hline Age (Years) & -0.02 & 0.00 & 0.98 & 0.98 & 0.98 & .000 & -0.02 & 0.00 & 0.98 & 0.98 & 0.98 & .000 \\
\hline Time (Year) & 0.00 & 0.00 & 1.00 & 1.00 & 1.00 & .639 & 0.00 & 0.00 & 1.00 & 1.00 & 1.01 & .000 \\
\hline Educational Attainment (0-20) & 0.04 & 0.00 & 1.04 & 1.04 & 1.05 & .000 & 0.05 & 0.00 & 1.05 & 1.04 & 1.06 & .000 \\
\hline Fulltime Employment & 0.77 & 0.04 & 2.17 & 2.01 & 2.33 & .000 & 0.73 & 0.03 & 2.09 & 1.95 & 2.23 & .000 \\
\hline Marital Status (Married) & 0.22 & 0.04 & 1.24 & 1.15 & 1.33 & .000 & 0.36 & 0.03 & 1.44 & 1.35 & 1.53 & .000 \\
\hline Constant & 1.49 & 0.07 & 4.45 & & & .000 & 1.26 & 0.06 & 3.52 & & & .000 \\
\hline
\end{tabular}

CI: Confidence Interval; SE: Standard Error;

OR: Odds Ratio

Table 5. Summary of logistic regressions on happiness in men and women.

\begin{tabular}{|c|c|c|c|c|c|c|c|c|c|c|c|c|}
\hline \multirow[b]{3}{*}{ Race/ethnicity (Black) } & \multicolumn{6}{|c|}{ Men } & \multicolumn{6}{|c|}{ Women } \\
\hline & \multirow{2}{*}{$\begin{array}{c}b \\
-0.43 \\
\end{array}$} & \multirow{2}{*}{$\begin{array}{c}\text { SE } \\
0.05\end{array}$} & \multirow{2}{*}{$\begin{array}{c}\text { OR } \\
0.65\end{array}$} & \multicolumn{2}{|c|}{$95 \% \mathrm{Cl}$} & \multirow{2}{*}{$\begin{array}{c}\mathrm{p} \\
.000\end{array}$} & \multirow{2}{*}{$\begin{array}{c}\mathrm{b} \\
-0.48 \\
\end{array}$} & \multirow{2}{*}{$\begin{array}{c}\text { SE } \\
0.04\end{array}$} & \multirow{2}{*}{$\begin{array}{c}\text { OR } \\
0.62\end{array}$} & \multicolumn{2}{|c|}{$95 \% \mathrm{Cl}$} & \multirow{2}{*}{$\begin{array}{c}\mathrm{P} \\
.000\end{array}$} \\
\hline & & & & 0.59 & 0.72 & & & & & 0.57 & 0.68 & \\
\hline Race/ethnicity (Other) & -0.16 & 0.08 & 0.85 & 0.72 & 0.99 & .042 & -0.36 & 0.07 & 0.70 & 0.61 & 0.81 & .000 \\
\hline Age (Years) & 0.00 & 0.00 & 1.00 & 1.00 & 1.00 & .361 & 0.00 & 0.00 & 1.00 & 1.00 & 1.00 & .055 \\
\hline Time (Year) & 0.00 & 0.00 & 1.00 & 1.00 & 1.01 & .170 & 0.00 & 0.00 & 1.00 & 1.00 & 1.00 & .494 \\
\hline Educational Attainment (0-20) & 0.03 & 0.00 & 1.04 & 1.03 & 1.04 & .000 & 0.03 & 0.00 & 1.03 & 1.03 & 1.04 & .000 \\
\hline Fulltime Employment & 0.57 & 0.04 & 1.77 & 1.63 & 1.92 & .000 & 0.38 & 0.04 & 1.46 & 1.36 & 1.57 & .000 \\
\hline Marital Status (Married) & 0.87 & 0.04 & 2.38 & 2.20 & 2.59 & .000 & 0.98 & 0.04 & 2.66 & 2.47 & 2.86 & .000 \\
\hline Constant & 0.92 & 0.08 & 2.52 & & & .000 & 1.20 & 0.07 & 3.32 & & & .000 \\
\hline
\end{tabular}

$\mathrm{CI}$ : Confidence Interval; SE: Standard Error;

OR: Odds Ratio

\section{Discussion}

We found that full-time employment and marriage may be more closely associated with happiness for American men and women, respectively. Regarding determinants of subjective health, we found educational attainment and marital status to be more salient for American women than American men. Thus, gender alters the salience of social determinants of subjective health and happiness, two key indicators of wellbeing of Americans.

White race, female gender, young age, high educational attainment, being married, and having a full-time job were associated with better subjective health and happiness. Other studies have shown that subjective health and happiness, two key elements of human well-being, are affected by educational level ${ }^{7,32,33}$, marital status ${ }^{7,33,34}$, and income $\mathrm{e}^{7,32-35}$. Other determinants of these measures include gender $7,32,33,36$, age ${ }^{13,32-34}$, race $^{37-40}$, culture ${ }^{34,41}$, social support ${ }^{7,34}$, and even genetics ${ }^{36,42}$. Due to a genetic predisposition, some people are happier than others. A study on identical twins who were brought up in different families showed that $35-50 \%$ of happiness is a result of nurture $^{36}$. Subjective health and happiness both predict mortality risk ${ }^{31}$.

American women report higher subjective health and happiness than American men. Despite a higher burden of psychological disorders ${ }^{43}$, women are more likely to report being happy ${ }^{42}$. These may be due to social or biological differences ${ }^{42}$. Regarding subjective health (SRH), most studies are in line with our findings regarding lowerrated health status in men $^{32,36,42}$; however, there are also a few studies in which men have reported better subjective health than women ${ }^{41,44}$. Subjective health shows cultural variation $^{43}$. Availability of positive social relations and social support may increase subjective health of men and women ${ }^{44}$.

Additionally, we found that age was negatively associated with subjective health. Worse subjective health of older people seems intuitive because aging is associated with chronic disease and health problems ${ }^{45}$. High age is also linked to worsening disability ${ }^{46}$. But how much a person is satisfied with their aging is important: successful aging may be associated with higher subjective health regardless of chronological age ${ }^{13}$.

As shown by other studies, marriage is associated with higher subjective health ${ }^{1,32,41,44,47,48}$. We also found that married participants claim better health status than others. Marriage is accompanied by better support, which may improve subjective health ${ }^{32,44}$. A high sense of trust may be another factor explaining why married men and women report better subjective health ${ }^{48}$. A trusting attitude toward the world results in high social capital and consequently, healthy and happy feelings ${ }^{49-51}$. Another mechanism could be financial issues. Unmarried people (never married, divorced, or widowed) tend to face more economic 
problems than married people ${ }^{48}$. Besides, divorce has many detrimental effects on individual health and happiness ${ }^{48}$.

Considering the gender differences, in line with other studies $^{48}$, marital status had a more significant effect on women's health compared to men. Financial and psychological problems may be more prominent in the life of widowed women compared to their male counterparts ${ }^{48}$. In contrast, Chung et al. showed that married women in Eastern Asian countries report poorer subjective health than married men, which may be due to differences between Western and Eastern cultures ${ }^{41}$. Social networks are crucial in feeling happy, and marriage has a remarkable effect on the composition of social network and social relations, particularly for $\mathrm{men}^{34}$. However, marital satisfaction is an essential factor which determines how marital status impacts subjective health and happiness ${ }^{41,47}$.

As mentioned above, we found marriage to be more salient in shaping American women's happiness compared to American men. This seems true for many Western countries but not necessarily all countries, as opposite patterns are reported from some East Asian countries ${ }^{34}$. Cultural issues may interfere with the happiness of married men and women compared to that of single men and women $^{34}$. In a study on 500 young Iranians, participants did not mention marital status as an important factor for happiness ${ }^{52}$.

In line with a study conducted in Sweden ${ }^{48}$, we found that full-time employment had a larger effect on subjective health for men than for women. It is unknown if this is because men and women have different types of jobs. Compared to women, men are more likely to be selfemployed, do skilled manual labor, and have a high rank in their institutions and organizations ${ }^{48}$. Another reason may be gendered roles. Globally, it is expected from men to support their families and be the breadwinners; however, this pattern is more crucial in more traditional societies ${ }^{53}$. It is not only having a job, but having a secure job which is an important determinant of subjective health and happiness ${ }^{35}$.

We found major gender differences in the strength of effects of having a full-time job and marriage on happiness. While having a full-time job was a salient indicator of happiness in American men, marriage played a more pivotal role in the happiness of American women. The positive association between working full-time and happiness in men has a robust consistency regardless of the study population and region ${ }^{34}$. Having a full-time job brings economic prosperity which is essential for happiness ${ }^{34}$. Employment also extends the social network, another critical factor positively associated with happiness ${ }^{34}$. We argue that having a full-time job is a major determinant of the subjective health and happiness among American men.
The association between educational attainment and subjective health is well-established ${ }^{40}$. We found that education may have a more prominent effect in shaping women's than men's subjective health. Our finding was supported by several studies ${ }^{54-56}$. Higher education is associated with low disability ${ }^{46}$, improved subjective health and well-being ${ }^{20}$, better sleep quality ${ }^{26}$, and decreased mental distress and depressive symptoms ${ }^{23}$. However, advantages related to higher educational attainment, such as longer life expectancy, may depend on demographic factors such as gender and race ${ }^{17}$. A study conducted in six European countries showed that education had a positive correlation with subjective health in older-aged men; this was because increased education led to improved working conditions for men but not for women ${ }^{57}$.

The effect of race was also significant for happiness and subjective health, with Whites reporting greater health and happiness compared to other ethnic groups. This is not an uncommon finding as minority populations (e.g., Blacks, Asian Americans, etc.) rate their health and happiness lower than Whites ${ }^{58}$. Racial and ethnic groups including Blacks have suffered economic disadvantages due to historical injustice, racism, and discrimination ${ }^{16,39}$.

Countries and cultures vary in their levels of happiness. Some research has suggested that Western countries may be happier compared to Eastern countries ${ }^{59}$. Of all countries in North and South America, Mexico had the highest happiness score (8.3 out of 10), more than the USA (7.1) and Canada (7.8). This stands in contrast with the happiness score of many East Asian countries, e.g., Japan (6.1), China (6.5), and South Korea (6.1) ${ }^{59}$. These patterns may also reflect cultural variation in the expression of emotions as well as response style. While American culture admires exceptionalism, differences, and expression of extreme feelings, Asian culture values moderate states and devalues extreme emotions including extreme happiness ${ }^{60,61}$. As a result, Americans are more likely than Asian people to select an extreme response to a happiness question $^{60-62}$.

Countries also vary widely in the effects of social determinants of subjective health. This is demonstrated by the Research on Early Life and Aging Trends and Effects (RELATE) study, which included 9,179 adults with diabetes from 15 countries, namely, the United States, Costa Rica, India, Ghana, China, Puerto Rico, Mexico, Barbados, Brazil, Chile, Cuba, Uruguay, South Africa, Argentina, and Russia. The study documented major cross-country differences in the additive effects of socio-economic characteristics, health behaviors and comorbidities on subjective health of patients with diabetes. No social determinant of health showed a universal effect on subjective health of patients with diabetes. Heart disease was the only universal determinant of poor subjective health in all countries ${ }^{63}$. 
In another study that used RELATE data, higher education and income were associated with lower disability in only $31 \%$ and $23 \%$ of the countries, respectively. Gender and smoking showed inconsistent results; however, the number of comorbid medical conditions and age were determinants of disability in $85 \%$ of the countries ${ }^{46}$. The RELATE study suggests that each country shows a unique profile of social and behavioral determinants of subjective health. As a result, universal programs that are based on the belief that determinants of well-being are similar across different countries may be over-simplistic and produce less-thanexpected effects. Instead of universal programs, countries should implement locally designed interventions that are based on local data ${ }^{46,63-66}$. Our findings are only relevant to the USA. More cross-country research is needed on the topic.

\section{Strength and limitations}

The design of this study was cross-sectional, which does not permit causal inferences between the factors and the response variables (subjective health and happiness). Another weakness of this study was the lack of data regarding the quality of employment or of marital life ${ }^{41,47}$. The results of this study may not be similarly relevant to all age groups ${ }^{67}$. Future research may compare the relevance of social determinants on health and happiness of youth and older groups. However, this study is unique in that it was based on a large, nationally representative sample of Americans. Additionally, we included three leading SES indicators, namely, education, employment, and marital status, which affect the happiness and health of societies. Furthermore, this study extends the existing literature on gender difference in happiness and subjective health. Few studies have focused on this subject, especially in the US.

\section{Conclusion}

In the United States, drivers of subjective health and happiness may differ for men and women. While having a full-time job may bring more happiness to men, marriage plays a more salient role in shaping happiness for women. At the same time, high educational attainment and marriage may be more important for the subjective health of American women than for men. As social determinants of well-being vary across cultures and countries, these data help US policymakers who require local and intersectional data on the drivers of well-being of various subsections of the population.

\section{Funding}

The research reported in this publication was supported by the National Institutes of Health (NIH) awards 5S21MD000103, 54MD008149, R25 MD007610, 2U54MD007598, and U54 TR001627.

\section{Conflicts of Interest}

The authors declare no conflict of interest.

\section{Author Contributions}

S.A. conceptualized the study, analyzed the data, and prepared the first draft of the paper. N.M. drafted and revised the paper. M.B. and S.C. contributed to the conceptualization of the paper and revised the paper. All authors approved the final draft.

\section{References}

1. Subramanian SV, Kim D, Kawachi I. Covariation in the socioeconomic determinants of self rated health and happiness: a multivariate multilevel analysis of individuals and communities in the USA. J Epidemiol Community Health. 2005; 59: 664-669. doi:10.1136/ jech.2004.025742.

2. Fleche S, Smith C, Sorsa P. Exploring determinants of subjective wellbeing in OECD countries. 2012.

3. Joshi U. Subjective well-being by gender. Journal of Economics and Behavioral Studies. 2010; 1: 20-26.

4. DeSalvo KB, Jones TM, Peabody J, et al. Health care expenditure prediction with a single item, self-rated health measure. Medical care. 2009; 440-447.

5. Huppert F. Happiness breeds prosperity. Nature. 2010; 464: 12751276.

6. Böckerman P, Bryson A, Viinikainen J, et al. The biometric antecedents to happiness. PloS one. 2017; 12: e0184887, doi:10.1371/journal. pone. 0184887.

7. Azizi M, Mohamadian F, Ghajarieah M, et al. The Effect of Individual Factors, Socioeconomic and Social Participation on Individual Happiness: A Cross-Sectional Study. J Clin Diagn Res. 2017; 11: VC01VC04. doi:10.7860/JCDR/2017/24658.9982.

8. Barak Y. The immune system and happiness. Autoimmunity reviews. 2006; 5: 523-527. doi:10.1016/j.autrev.2006.02.010.

9. Zhang D, Chan DC, Niu L, et al. Meaning and its association with happiness, health and healthcare utilization: A cross-sectional study. Journal of affective disorders. 2018; 227: 795-802.

10. Bellis MA, Hughes $\mathrm{K}$, Jones $\mathrm{A}$, et al. Childhood happiness and violence: a retrospective study of their impacts on adult well-being. BMJ Open. 2013; 3: e003427, doi:10.1136/bmjopen-2013-003427.

11. Assari S. Unequal Gain of Equal Resources across Racial Groups. Int J Health Policy Manag. 2018; 7: 1-9. doi:10.15171/ijhpm.2017.90.

12. Maharlouei N, Akbari M, Khabbaz Shirazy M, et al. Factors associated with self-rated health status in Southwestern Iran: a populationbased study. Public health. 2016; 140: 179-185.

13. Whitley E, Popham F, Benzeval M. Comparison of the Rowe-Kahn model of successful aging with self-rated health and life satisfaction: The West of Scotland Twenty-07 prospective cohort study. The Gerontologist. 2016; 56: 1082-1092.

14. Idler EL, Benyamini Y. Self-rated health and mortality: a review of twenty-seven community studies. Journal of health and social behavior. $1997 ; 21-37$.

15. Berchick ER, Lynch SM. Regional variation in the predictive validity of self-rated health for mortality. SSM - Population Health. 2017; 3: 275 282, doi:10.1016/j.ssmph.2017.01.010.

16. Assari S, Caldwell CH. Mental Health Service Utilization among Black Youth; Psychosocial Determinants in a National Sample. Children (Basel). 2017; 4. doi:10.3390/children4050040. 
17. Assari S. Life Expectancy Gain Due to Employment Status Depends on Race, Gender, Education, and Their Intersections. J Racial Ethn Health Disparities. 2018; 5: 375-386. doi:10.1007/s40615-017-0381-x.

18. Assari S. Socioeconomic Status and Self-Rated Oral Health; Diminished Return among Hispanic Whites. Dent J (Basel). 2018; 6. doi:10.3390/ dj6020011.

19. Assari S, Caldwell CH, Bazargan M. Association Between Parental Educational Attainment and Youth Outcomes and Role of Race/ Ethnicity. JAMA Netw Open. 2019; 2: e1916018. doi:10.1001/ jamanetworkopen.2019.16018.

20. Assari S, Bazargan M. Educational Attainment and Subjective Health and Well-Being; Diminished Returns of Lesbian, Gay, and Bisexual Individuals. Behavioral Sciences. 2019; 9: 90.

21. Assari S. Ethnic and Gender Differences in Additive Effects of Socio-economics, Psychiatric Disorders, and Subjective Religiosity on Suicidal Ideation among Blacks. Int J Prev Med. 2015; 6: 53. doi:10.4103/2008-7802.158913.

22. Assari S. Social Determinants of Depression: The Intersections of Race, Gender, and Socioeconomic Status. Brain Sci. 2017; 7. doi:10.3390/ brainsci7120156.

23. Assari S. Combined Racial and Gender Differences in the Long-Term Predictive Role of Education on Depressive Symptoms and Chronic Medical Conditions. J Racial Ethn Health Disparities. 2017; 4: 385396. doi:10.1007/s40615-016-0239-7.

24. Assari S, Caldwell CH, Mincy R. Family Socioeconomic Status at Birth and Youth Impulsivity at Age 15; Blacks' Diminished Return. Children (Basel). 2018; 5. doi:10.3390/children5050058.

25. Assari S, Moghani Lankarani M. Race and Gender Differences in Correlates of Death Anxiety Among Elderly in the United States. Iran Psychiatry Behav Sci. 2016; 10: e2024. doi:10.17795/ijpbs-2024.

26. Assari S, Moghani Lankarani M, Kazemi Saleh D, et al. Gender modifies the effects of education and income on sleep quality of the patients with coronary artery disease. Int Cardiovasc Res J. 2013; 7: 141-146.

27. Assari S, Nikahd A, Malekahmadi MR, et al. Race by Gender Group Differences in the Protective Effects of Socioeconomic Factors Against Sustained Health Problems Across Five Domains. J Racial Ethn Health Disparities. 2016. 10.1007/s40615-016-0291-3, doi:10.1007/ s40615-016-0291-3.

28. Assari S, Smith J, Bazargan M. Health-Related Quality of Life of Economically Disadvantaged African American Older Adults: Age and Gender Differences. Int J Environ Res Public Health. 2019; 16. doi:10.3390/ijerph16091522.

29. Lankarani MM, Shah S, Assari S. Gender Differences in Vulnerability to Socioeconomic Status on Self-Rated Health in 15 Countries. Womens Health Bull. 2017; 4: e45280.

30. Assari S, Smith J, Bazargan M. Depression Fully Mediates the Effect of Multimorbidity on Self-Rated Health for Economically Disadvantaged African American Men but Not Women. Int J Environ Res Public Health. 2019. 16. doi:10.3390/ijerph16101670.

31. Assari S. Gender differences in the predictive role of self-rated health on short-term risk of mortality among older adults. SAGE Open Med. 2016; 4: 2050312116666975. doi:10.1177/2050312116666975.

32. Xu W, Sun H, Zhu B, et al. Analysis of Factors Affecting the High Subjective Well-Being of Chinese Residents Based on the 2014 China Family Panel Study. 2019; 16. doi:10.3390/ijerph16142566.

33. Miret M, Caballero FF, Chatterji S, et al. Health and happiness: crosssectional household surveys in Finland, Poland and Spain. Bulletin of the World Health Organization. 2014; 92: 716-725. doi:10.2471/ blt.13.129254.

34. Hori M, Kamo Y. Gender differences in happiness: The effects of marriage, social roles, and social support in East Asia. Applied Research in Quality of Life. 2018; 13: 839-857.
35. Barrech A, Baumert J, Gündel H, et al. The impact of job insecurity on long-term self-rated health - results from the prospective populationbased MONICA/KORA study. BMC public health. 2018; 18: 754, doi:10.1186/s12889-018-5621-4.

36. Bartels M1, Saviouk V, de Moor MH, et al. Heritability and genomewide linkage scan of subjective happiness. Twin Research and Human Genetics. 2010; 13: 135-142.

37. Akinhanmi M01, Biernacka JM2,3, Strakowski SM, et al. Racial disparities in bipolar disorder treatment and research: a call to action. Bipolar Disord. 2018; 20: 506-514, doi:10.1111/bdi.12638.

38. Assari S, Caldwell CH, Mincy RB. Maternal Educational Attainment at Birth Promotes Future Self-Rated Health of White but Not Black Youth: A 15-Year Cohort of a National Sample. J Clin Med. 2018; 7. doi:10.3390/jcm7050093.

39. Assari S, Preiser B. Education and Income Predict Future Emotional Well-Being of Whites but Not Blacks: A Ten-Year Cohort. 2018; 8. doi:10.3390/brainsci8070122.

40. Assari, S. Race, Education Attainment, and Happiness in the United States. International journal of epidemiologic research. 2019; 6: 76.

41. Chung W, Kim R. Are Married Men Healthier than Single Women? A Gender Comparison of the Health Effects of Marriage and Marital Satisfaction in East Asia. PloS one. 2015; 10: e0134260. doi:10.1371/ journal.pone. 0134260 .

42. Chen H, Pine DS, Ernst M, et al. The MAOA gene predicts happiness in women. Progress in neuro-psychopharmacology \& biological psychiatry. 2013; 40:122-125. doi:10.1016/j.pnpbp.2012.07.018.

43. Baxter AJ, Vos T, Scott KM, et al. The global burden of anxiety disorders in 2010. Psychological medicine. 2014; 44: 2363-2374.

44. Von dem Knesebeck O, Geyer S. Emotional support, education and self-rated health in 22 European countries. BMC public health. 2007; 7: 272

45. Prasad S, Sung B, Aggarwal BB. Age-associated chronic diseases require age-old medicine: role of chronic inflammation. Prev Med. 2012; 54 Suppl: S29-37. doi:10.1016/j.ypmed.2011.11.011.

46. Assari S. Cross-Country Differences in the Additive Effects of Socioeconomics, Health Behaviors and Medical Comorbidities on Disability among Older Adults with Heart Disease. J Tehran Heart Cent. 2015; 10: 24-33.

47. Chung W, Kim R. Does marriage really matter to health? Intraand inter-country evidence from China, Japan, Taiwan, and the Republic of Korea. PloS one. 2014; 9: e104868. doi:10.1371/journal pone. 0104868

48. Lindstrom M. Marital status, social capital, material conditions and self-rated health: a population-based study. Health policy (Amsterdam, Netherlands). 2009; 93: 172-179. doi:10.1016/j. healthpol.2009.05.010.

49. Lindstrom M. Does social capital include trust? Commentary on Carpiano and Fitterer (2014). Soc Sci Med. 2014; 116: 235-236, doi:10.1016/j.socscimed.2014.04.028

50. Cozzolino PJ. Trust, cooperation, and equality: a psychological analysis of the formation of social capital. Br J Soc Psychol. 2011; 50: 302-320. doi:10.1348/014466610X519610

51. Mohseni M, Lindstrom M. Social capital, political trust and self ratedhealth: a population-based study in southern Sweden. Scand J Public Health. 2008; 36: 28-34. doi:10.1177/1403494807085078.

52. Mehrdadi A, Sadeghian S, Direkvand-Moghadam A, et al. Factors Affecting Happiness: A Cross-Sectional Study in the Iranian Youth. Journal of clinical and diagnostic research : JCDR. 2016; 10: Vc01vc03. doi:10.7860/jcdr/2016/17970.7729. 
53. Marks J, Bun LC, McHale SM. Family Patterns of Gender Role Attitudes. Sex Roles. 2009; 61: 221-234. doi:10.1007/s11199-009-9619-3.

54. Liu H, Hummer RA. Are educational differences in U.S. self-rated health increasing?: an examination by gender and race. Social science \& medicine (1982). 2008; 67: 1898-1906. doi:10.1016/j. socscimed.2008.09.021.

55. Pinillos-Franco S, García-Prieto C. The gender gap in self-rated health and education in Spain. A multilevel analysis. PloS one. 2017; 12.

56. Assari S, Cobb S, Bazargan M. Race by Gender Differences in the Protective Effects of Education and Income Against Subsequent Changes in Self-rated Health, Physical Activity, and Body Mass Index Among Older Americans. Journal of health economics and development. 2019; 1: 9-21.

57. Mazzonna F. The long lasting effects of education on old age health: evidence of gender differences. Social Science \& Medicine. 2014; 101: 129-138.

58. Weaver CN. Happiness of Asian Americans. Psychol Rep. 2003; 93: 1032-1034. doi:10.2466/pr0.2003.93.3f.1032.

59. Veenhoven R. World database of happiness. Erasmus University Rotterdam, Happiness Economics Research Organization: 2014.

60. Chen C, Lee Sy, Stevenson HW. Response style and cross-cultural comparisons of rating scales among East Asian and North American students. Psychological Science. 1995; 6: 170-175.
61. Uchida Y, Norasakkunkit V, Kitayama S. Cultural constructions of happiness: theory and emprical evidence. Journal of happiness studies. 2004; 5: 223-239.

62. Kitayama S, Park J. Cultural neuroscience of the self: Understanding the social grounding of the brain. Social cognitive and affective neuroscience. 2010; 5: 111-129.

63. Assari S. Cross-country variation in additive effects of socioeconomics, health behaviors, and comorbidities on subjective health of patients with diabetes. J Diabetes Metab Disord. 2014; 13: 36. doi:10.1186/2251-6581-13-36.

64. Assari S, Lankarani MM. Does Multi-morbidity Mediate the Effect of Socioeconomics on Self-rated Health? Cross-country Differences. Int J Prev Med. 2015; 6: 85. doi:10.4103/2008-7802.164413.

65. Assari S, Lankarani RM, Lankarani MM. Correction: Cross-country differences in the association between diabetes and disability. J Diabetes Metab Disord. 2014; 13: 73. doi:10.1186/2251-6581-13-73.

66. Assari S, Lankarani RM, Lankarani MM. Cross-country differences in the association between diabetes and disability. I Diabetes Metab Disord. 2014; 13: 3. doi:10.1186/2251-6581-13-3.

67. Dowd JJ, Bengtson VL. Aging in minority populations. An examination of the double jeopardy hypothesis. J Gerontol. 1978; 33: 427-436. 